\title{
Análisis espacial para la prevención de riesgo de inundación en la colonia Encinal, Xicotepec
}

\author{
Spatial analysis for flood risk prevention \\ In the informal settlement Encinal, Xicotepec
}

\author{
Barrios-Rodríguez,Uriel ${ }^{1}$ \\ Salgado-Montes, Stephanie Scherezada ${ }^{2}$ \\ Flores-Lucero, María de Lourdes ${ }^{3}$ \\ ${ }^{1-3}$ Benemérita Universidad Autónoma de Puebla, Puebla, México \\ ${ }^{1}$ uriel.barrios@alumno.buap.mx/https://orcid.org/0000-0002-8705-2369 \\ ${ }^{2}$ stephanie.salgado@correo.buap.mx / https://orcid.org/0000-0001-6756-428X \\ 33maria.flores@correo.buap.mx/https://orcid.org/0000-0003-3849-0060
}

Recibido el 02 de octubre de 2021, aprobado el 07 de diciembre de 2021

RESUMEN | Las inundaciones en asentamientos irregulares, que ocasionan pérdidas humanas, bienes y problemas sanitarios, son consecuencia de la ocupación antrópica al margen de la planificación territorial y de las prácticas permisivas que autorizan la construcción de viviendas en suelos no aptos para el desarrollo urbano. Ejemplo de ello es el caso de la colonia Encinal en el municipio de Xicotepec; asentamiento irregular ubicado en la periferia de la cabecera municipal, cuya morfología responde a pendientes escarpadas y el cauce de un arroyo, este último degradado a canal de aguas residuales, presenta desbordamiento en época de lluvias torrenciales. Las inundaciones colocan en condición de alto riesgo a 230 habitantes y 49 viviendas. La carencia de información y análisis de riesgo limita la identificación de las condiciones que inciden en la construcción social del riesgo de inundación, la vulnerabilidad física y social, ya que los Sistemas de Información Geográfica (SIG) se han subutilizado como tecnología para el análisis espacial de riesgos de desastre. El objetivo de esta investigación es demostrar la relevancia de la tecnología de los SIG en el análisis socioespacial del proceso de construcción del riesgo en un asentamiento irregular. La metodología es cuantitativa y cualitativa; se aplicaron los SIG para la georreferenciación y el procesamiento de datos, y la elaboración de cartografía para la representación de los resultados del análisis espacial. Una conclusión relevante es que, los SIG son una tecnología que permite generar información detallada para evidenciar las condiciones de vulnerabilidad en asentamientos irregulares, y coadyuvar a la toma de decisiones bajo el enfoque de prevención y mitigación del riesgo de desastre.

PALABRAS CLAVE | Sistemas de información geográfica, análisis espacial, riesgo de inundación, vulnerabilidad, asentamiento irregular

ABSTRACT | Floods in informal settlements that cause human and economic losses and health problems are consequence of anthropic occupation outside of territorial planning, and permissive 
practices that authorize the construction of houses on land unsuitable for urban development. An example of this is the case of the Encinal in the municipality of Xicotepec, Puebla, which is an informal settlement located on the periurban area, whose morphology responds to steep slopes and the bed of a stream, the latter has been degraded to a sewage canal and overflows during the months of June to October. Therefore, 230 inhabitants and 49 homes have been identified in a high-risk flood zone. The lack of geographic and statistical information is a limitation to analyze the anthropic conditions that influence the construction of the flood risk, as well as identify physical and social vulnerability, in this sense, Geographic Information Systems (GIS) have been underused as a technology for disaster risk spatial analysis. The objective of this research is to demonstrate the relevance of GIS in the flood risk construction process analysis in an irregular settlement. In the quantitative and qualitative methodology, GIS was applied as a technology for spatial analysis, georeferencing of data in field trips, and mapping for the representation of flood risk conditions in the Encinal settlement. A relevant conclusion is that GIS is a technology that allows generating detailed information to show the situation of vulnerability in informal settlements and contribute to decision making to prevent and mitigate disaster risk.

KEYWORD | Bodily experience, process of quotidianization, everyday space, extended proprioception, phenomenology

\section{Introducción}

La construcción social del riesgo define el proceso en el cual diferentes fenómenos naturales se han transformado en peligros socialmente inducidos debido a la fuerte transformación y degradación de la naturaleza, la explotación de recursos naturales, las relaciones interdependientes que presentan con amenazas de tipo contaminante o tecnológico (Lavell, 1996; Beck, 1998), y con condiciones de vulnerabilidad (Merlinsky \& Tobías, 2016).

La generación de riesgos es una consecuencia de la transformación de la naturaleza por las personas para la construcción de hábitats como los asentamientos urbanos (Ríos-Llamas, 2020). En este sentido las personas pueden y deben desarrollar capacidades para prevenir y mitigar el riesgo, desarrollar la resiliencia y adaptación de dichos asentamientos y los habitantes.

En la gestión del riesgo de desastre, los individuos tienen un rol fundamental para desarrollar acciones proactivas (Mojtahedi \& Oo, 2016), ya que esta refiere a "un proceso social cuyo fin último es la previsión, la reducción y el control permanente de los factores de riesgo de desastre [...] en consonancia con, e integrada al logro de pautas de desarrollo humano, económico, ambiental y territorial, sostenibles" (Narvéz, Lavell, \& Pérez, 2009).

Los problemas detectados en la gestión del riesgo son la carencia de acciones proactivas que incluyan la predicción y prevención, colaboración entre instituciones de diferentes niveles, la participación local e internacional de Organizaciones no Gubernamentales (ONG), instrumentos de planeación, y se resalta, la carencia de educación, conocimiento, información y bases de datos (Moe \& Pathranarakul, 2006).

El análisis espacial y los Sistemas de Informaición Geográfica (SIG) permiten explorar y explicar los procesos sociales y espaciales que inciden en la contrucción del riesgo y la vulnerabilidad en 
asentamientos urbanos para cuantificar y caracterizar variables mediante el análisis de distribución y multivariable (Narvaéz, Durán, Menoscal, \& Bayón, 2020; Principi \& Buzai, 2021).

El análisis exploratorio y comparativo de datos georreferenciados de zonas de residencia y equipamientos, la distancia respecto a los cauces, tipos de suelo, pendiente del terreno y precipitaciones, así como el acercamiento cartográfico para la interpretación modelística sirven para definir con precisión los niveles de riesgo de inundación y vulnerabilidad (Narvéz, Lavell, \& Pérez, 2009).

En México, la gestión integral de riesgos de desastres ha progresado a través de una política pública (Gobierno federal, 2021) adoptada por acuerdos internacionales (ONU, 2015) y su incorporación en instrumentos como planes de desarrollo, programas de ordenamiento territorial y atlas de riesgos, sin embargo, carecen de sustento metodológico e información precisa que fundamenten los diagnósticos y estrategias.

Algunos instrumentos contienen información en escalas estatales y municipales desactualizadas que limita el análisis y el planteamiento de estrategias en asentamientos urbanos, y aún menos en asentamientos irregulares ${ }^{1}$, mismos que se consideran prioritarios a atender debido los altos niveles de vulnerabilidad que presentan por el rezago social y de vivienda (Hernández, Barrios, \& Ramírez, 2017; CONEVAL, 2021).

Si bien el marco legal cuenta con un enfoque preventivo, en la práctica las acciones son reactivas ante la emergencia y el desastre, situación que se ve potenciada en asentamientos irregulares carentes de estudios de riesgo que fundamenten estrategias para mitigarlo (Plot, 2011). Se identifican estas debilidades en el análisis del riesgo ya que no han logrado articular el enfoque sistémico y espacial (Buzai, 2009).

En los Atlas de Riesgos se aplica la tecnología de los SIG para el análisis de riesgos de inundación mediante herramientas y modelos matemáticos y gráficos (Omelio, 2012; Yamanaka Victor H, 2011), y para modelar las amenazas y riesgos en mapas digitales e interactivos en escala estatal y municipal (CENAPRED, 2009). No obstante, estas escalas no permiten identificar asentamientos urbanos, ni irregulares.

En el caso del municipio de Xicotepec en el Estado de Puebla, los desastres en las últimas 3 décadas se deben a fenómenos hidrometeorológicos, el $89 \%$ de las pérdidas económicas son asociadas a la presencia de huracanes, tormentas tropicales y lluvias intensas todo el año (Salas, 2019) que se ven potenciadas por la modificación antrópica del territorio.

El municipio cuenta con el Plan de Desarrollo Municipal (2018-2021) y se está elaborando un Programa de Protección Civil, respecto a este último, en el proceso de elaboración las autoridades responsables no han tenido acercamiento con la población, los habitantes desconocen dicho instrumento, para sustentar los diagnósticos y las estrategias (Becerra, 2021).

En esta investigación se plantea la revaloración de los SIG a través del análisis socioespacial para evidenciar el proceso de construcción social del riesgo de inundación aplicado en el asentamiento irregular, la colonia Encinal. El análisis proporcionó información geoespacial para determinar las causas de la construcción del riesgo, e identificar las condiciones de vulnerabilidad física y social.

El artículo está estructurado en tres apartados 1) la construcción social del riesgo en asentamientos

1 Los asentamientos irregulares se caracterizan por población de bajos recursos económicos, que, dadas sus necesidades de vivienda, se asientan sobre suelos inseguros y factibles a sufrir un desastre (Aguilar \& Escamilla, 2009). 
irregulares para definir el proceso y variables de vulnerabilidad, 2) los sistemas de información geográfica como tecnología para generar información que evidencie el riesgo de inundación, y 3) la vulnerabilidad en la colonia Encinal.

\section{Metodología}

En la investigación se aplicó una metodología que combina el análisis de datos cuantitativo y cualitativo de la información estadísticas y geográficas bajo el enfoque de los sistemas complejos para construir el problema desde un acercamiento espacial (García, 2006; Principi \& Buzai, 2021).

El método principal fue el análisis socioespacial a través de Sistemas de Información Geográfica (SIG) para realizar el diagnóstico urbano que permitió definir la zona de riesgo de inundación y las condiciones de vulnerabilidad física y social (Buzai, 2009).

La revisión de literatura especializada permitió explicar la teoría que define la construcción social del riesgo como un proceso de producción y reproducción de las condiciones de vulnerabilidad que definen y determinan la magnitud de los efectos ante la presencia de una amenaza natural (Acosta, 2005; Lavell, 1996; Beck, 1998), así como, las variables y niveles que constituyen el riesgo y la vulnerabilidad (Ríos-Llamas, 2020).

En el análisis espacial, se utilizaron datos vectoriales de la carta geoestadística urbana en escala 1:50 000 (INEGI, 2016), la carta topográfica en escala 1:20 000 y las corrientes agua en escala 1:250 000, el Atlas de Riesgos Estatal (CENAPRED, 2009), los datos georreferenciados en las visitas de campo de los niveles máximos de agua y curvas de nivel, así como, los datos estadísticos del Censo de Población y Vivienda (INEGI, 2010).

Los datos vectoriales y alfanuméricos de las variables del medio natural y físico, se procesaron con el software QGis 3.14 mediante herramientas de geoprocesamiento y modelado hídrico, se clasificaron los tramos cauce, se definieron zonas de alto riesgo, y se identificó a la población y las viviendas vulnerables por manzana. Los resultados de este análisis se muestran en mapas temáticos.

El diagnóstico se complementó con el análisis de los actores involucrados a través de entrevistas virtuales (debido a la pandemia del COVID-19) para identificar la percepción de los habitantes respecto al riesgo de inundación, así como las acciones que realizan para prevenir y mitigar el riesgo, o actuar ante situaciones de emergencia.

Asimismo, se realizaron entrevistas semiestructuradas a las autoridades gubernamentales para identificar el nivel de aplicación de los SIG para la elaboración de estudios de riesgo de desastre.

\section{Desarrollo}

\section{Construcción social del riesgo en asentamientos irregulares}

Los impactos de amenazas a nivel mundial generaron $46.32 \%$ más desastres en la segunda década del siglo XX respecto a la primera, y solo una diferencia de $3.39 \%$ respecto a los primeros diecisiete años del siglo XXI (Salgado, 2020). Las pérdidas, humanas, materiales y económicas, generadas por desastres, derivan de la incidencia de las actividades humanas para modificar el territorio (Pelling , 2005; Merlinsky \& Tobías, 2016) 
A partir de los estudios de riesgo generados en la década de los 70 (Mojtahedi \& Oo, 2016), se replanteó el enfoque del riesgo de desastre. En la declaración del Decenio Internacional para la Reducción de los Desastres Naturales (ONU, 1987), ya no se consideraron los desastres como fenómenos inevitables de la naturaleza sino como fenómenos susceptibles de prevención y, ciertamente, de reducción.

Este cambio de paradigma plantea que los desastres, originalmente atribuidos a fenómenos naturales, son generados por las prácticas humanas relacionadas con la degradación ambiental, el intensivo crecimiento demográfico, los procesos poco regulados de urbanización y el incremento de las desigualdades socioeconómicas en las diferentes escalas territoriales (Acosta, 2005).

Desde entonces, han surgido estudios y reflexiones de autores como Wilches-Chaux (1993), Lavell (1996) y Lungo (2005) bajo el enfoque de la construcción social del riesgo. Estos se intensificaron y profundizaron hasta alcanzar Marco de Sendai para la Reducción del Riesgo de Desastres 2015-2030 (ONU, 2015), actualmente, instrumento referente de la gestión de riesgos de desastre a nivel internacional.

Las evidencias empíricas de dichos estudios fundamentaron la conceptualización de las probabilidades de exposición a la ocurrencia de desastres de grupos con rezago social en materia de educación, salud, servicios públicos básicos y vivienda precaria (Wilches-Chaux, 1993; Cardona, 2003; CONEVAL, 2021)

En países en desarrollo, se desplegaron modelos alternativos para el estudio del riesgo de desastres que tenían como eje el análisis de la vulnerabilidad, mismos que ya evidenciaban las debilidades y omisiones institucionales y de participación social, situación que limita conciencia respecto al riesgo de desastre, así como la toma de decisiones concretas (Wilches-Chaux, 1993; Lavell, 1996).

En Latinoamérica, destacan los asentamientos irregulares en riesgo, ya que se observa la constante incorporación de tierras sin vocación urbana y la modificación de ecosistemas (Aguilar \& Escamilla, 2009); aunado a la carencia de información y estudios de riesgo, los bajos índices de educación, bajo acceso a servicios de salud, servicios públicos básicos, y vivienda precaria (Cardona, 2003)

En este sentido, el riesgo de desastre asociado a la configuración urbana y condiciones de rezago en los asentamientos irregulares son incisivos, debido a la inestabilidad, inseguridad, carencias y fragilidad en que se encuentran la población y las viviendas (Merlinsky \& Tobías, 2016).

Particularmente en México, las inundaciones son fenómenos frecuentes que ocasionan desastres (Salgado, 2020), y se agudizan en asentamientos irregulares que presentan viviendas autoconstruidas con materiales endebles, bajo acceso a servicios públicos básicos e ilegalidad en la tenencia de la tierra (Vázquez-Sanchez \& Méndez-Ramírez, 2011; Córtes , 2003; Plot, 2011).

Estas variables de vulnerabilidad física y social pueden ser analizadas para evidenciar la construcción social del riesgo de inundación en un asentamiento irregular; en este sentido, la tecnología de los Sistemas de Información Geográfica (SIG) es potencial, mediante software libre, tablas de atributos, datos vectoriales y raster, y métodos de análisis espacial para estudiar el riesgo de inundación.

\section{Sistemas de Información Geográfica, tecnología para analizar el riesgo de desastre}

El análisis socioespacial de la construcción social del riesgo a través de Sistemas de Información 
Geográfica (SIG) incluye el procesamiento de datos espaciales y la elaboración de cartografía digital que "permite contrastar los diferentes cambios socio-territoriales y, por tanto, deducir la evolución espacial de una región o localidad y las repercusiones humanas sobre el medio" (Ruiz, Savé, \& Herrera, 2013).

Los SIG no solo posibilitan aplicaciones técnicas, sino que tienen un importante componente teórico-metodológico que hacen posible mirar la realidad de una manera específica, las bases se encuentran en una postura racionalista que brinda la posibilidad de pensar en una construcción regional y en una postura cuantitativa para la modelización del riesgo de desastre (Buzai, 2009).

El procesamiento de datos estadísticos y geográficos y la generación de cartografía digital, mediante herramientas automatizadas, permiten reproducir la física de las inundaciones en diferentes escenarios e integrar los resultados en mapas temáticos (Hernández, Barrios, \& Ramírez, 2017).

La determinación del riesgo y vulnerabilidad por inundación requiere un enfoque complejo debido a los factores involucrados y su estado evolutivo (Hernández, Barrios, \& Ramírez, 2017). Se considera necesario incluir variables referentes a la demografía, topografía, hidrografía, demografía, morfología urbana representados en datos vectoriales y raster para generar modelos matematicos y gráficos (Buzai, 2009).

El avance de la tecnología e informática entorno a los SIG ha permitido caracterizar y representar las condiciones de riesgo de una forma integral (Hernández, Barrios, \& Ramírez, 2017), y su aplicación se considera fundamental para la toma de decisiones concretas en materia de planeación urbana y ordenamiento territorial (ONU, 2015; Principi \& Buzai, 2021).

En Latinoamérica, existen lenta y débil aplicación de los SIG en el análisis de riesgos de desastre (Díaz Delgado, Baro Juárez, \& Bedolla Lara, 2011), la mayoría de las aplicaciones documentadas refieren a investigaciones (Radicelli, Pomboza, Villacrés, \& Bodero, 2019), proyectos piloto (Rodríguez \& García, 2012) o no están totalmente implementadas como en el caso de los atlas de riesgo y los programas.

En México, los estudios desarrollan la generación y actualización de mapas de riesgo a través de los SIG (Yamanaka Victor H, 2011), la debilidad radica en la carencia de los métodos de análisis y las bases de datos sociales y espaciales de los instrumentos de gestión y planeación, como los atlas de riesgo municipales que en el caso del Estado de Puebla presenta un déficit del 82\%.

El problema se complejiza cuando incorporamos dificultades en los procesos de gestión administrativa tales como los largos procesos administrativa-política y burocráticos o la falta de disponibilidad de datos accesibles, oficiales y actualizados como el caso de los catastros municipales (Buzai, 2009).

En contraste, las mayores aportaciones se generan en estudios de grado (Laguno \& Bourguett, 2014), en Puebla encontramos casos significativos como los de Ramírez (2017) y Salgado (2020). Sin embargo, es necesario profundizar en los métodos de análisis espacial a través de los SIG para procesar variables del riesgo de inundación y avanzar en la gestión integral del riesgo desde un enfoque preventivo.

\section{Resultados}

Colonia Encinal, una zona de riesgo de inundación 
El municipio de Xicotepec, está ubicado en el noroeste del Estado de Puebla, en la provincia fisiográfica conformada por la Sierra Norte del Estado de Puebla y la Sierra Madre Oriental; mediante el análisis espacial se determinó que cuenta con una altitud que oscila entre los 300 a 1400 metros, un área de 312.3 km2 y 80,591 habitantes (INEGI, 2010).

Se localiza en las subcuencas hidrográficas Río San Marcos y Río Necaxa respectivamente, en donde existen múltiples corrientes de agua intermitentes y perennes como ríos, arroyos y arroyuelos. La precipitación anual es de $170 \mathrm{~mm}$ y el periodo de lluvias más intenso se presenta entre los meses de junio y octubre (Meteoblue, 2021), fenómenos que contribuyen en el aumento de caudales y flujos pluviales.

En el municipio se han generado inundaciones urbanas en asentamientos ubicados en las riberas de los cauces, tal es el caso del asentamiento irregular colonia Encinal en la ciudad de Xicotepec de Juárez (cabecera municipal) que cuenta con 1,056 habitantes y 286 viviendas (INEGI, 2010) (ver figura 1).

El asentamiento se construyó en la ribera ${ }^{2}$ de un arroyo que fue constreñido para la construcción de viviendas y rellenado con residuos sólidos, en el que se han registrado precipitaciones de 361 mm (Meteoblue, 2021) que ocasionan el desbordamiento de flujos e inundaciones. Además, el asentamiento se ubica a una distancia de dos metros aproximadamente de ductos de PEMEX y líneas de alta tensión.

El análisis espacial de las variables sociales y físicas permitieron estimar 230 habitantes y 49 viviendas en la zona de riesgo de inundación. En situaciones de emergencia, ante la acumulación de flujos pluviales y el colapso del drenaje, los habitantes deben evacuar las viviendas.

Figura 1. Localización del sitio

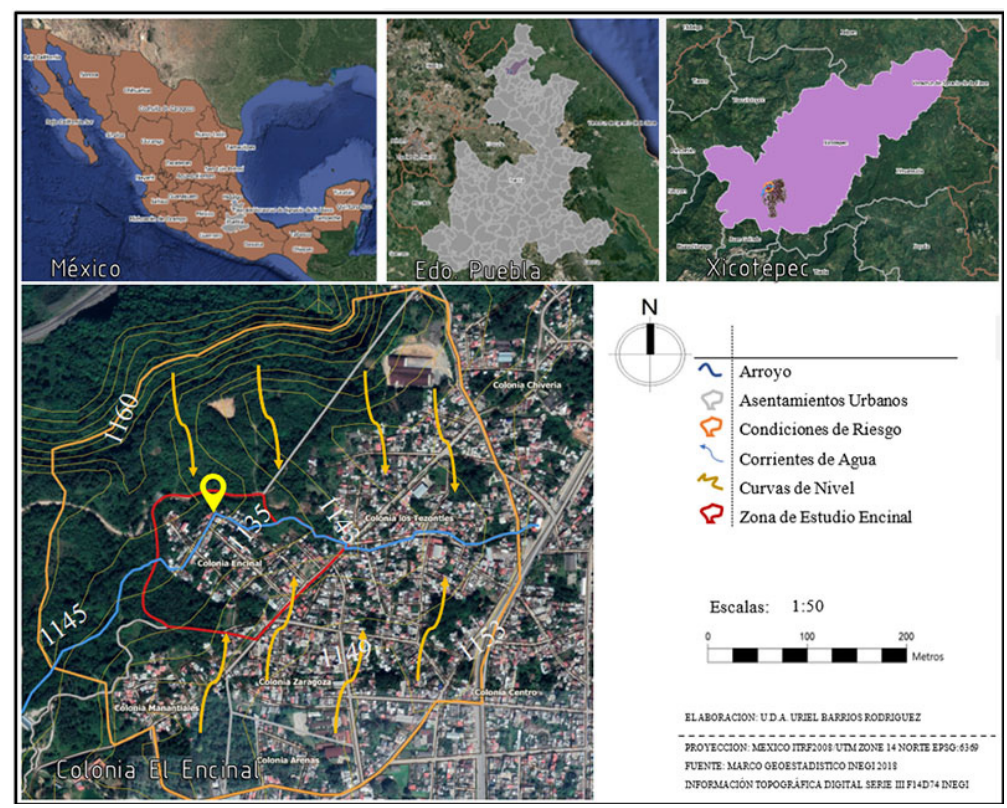

Nota. Elaborada por el autor

2 Ribera o Zona Federal son las fajas de diez metros de anchura contiguas al cauce de las corrientes o al vaso de los depósitos de propiedad nacional, medidas horizontalmente a partir del nivel de aguas máximas ordinarias. La amplitud de la ribera o zona federal será de cinco metros en los cauces con una anchura no mayor de cinco metros (CPEUM, 2020). 


\section{Un entorno con riqueza hídrica}

Las condiciones naturales de la colonia Encinal son determinantes, se ubica entre 1160 y 1132 metros de altitud (ver figura 2), presenta pendientes fuertes con inclinación del 18\%, deslizamiento de suelo y rocas ígneas que se depositan en el arroyo, los escurrimientos intermitentes tienen una precipitación anual de $170 \mathrm{~mm}$ (Meteoblue, 2021), y una intensidad de $218 \mathrm{~mm}$ a $361 \mathrm{~mm}$ entre junio y octubre (SMN, 2019) (ver figura 3).

Figura 2. Condiciones de riesgo

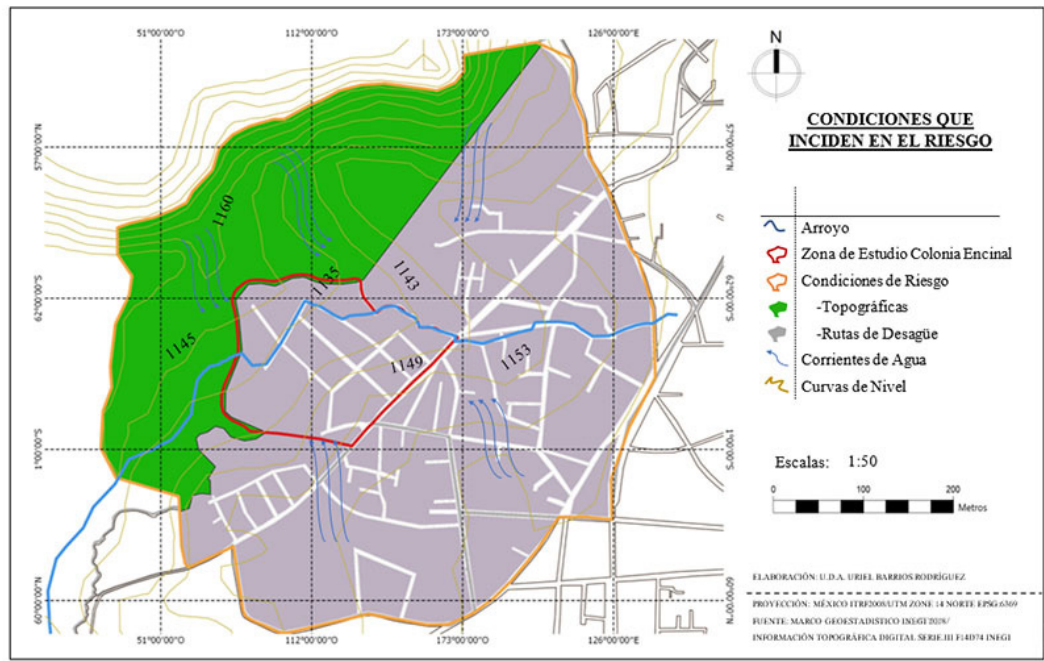

Nota. Elaborada por el autor

Figura 3. Precipitación y tempetarura

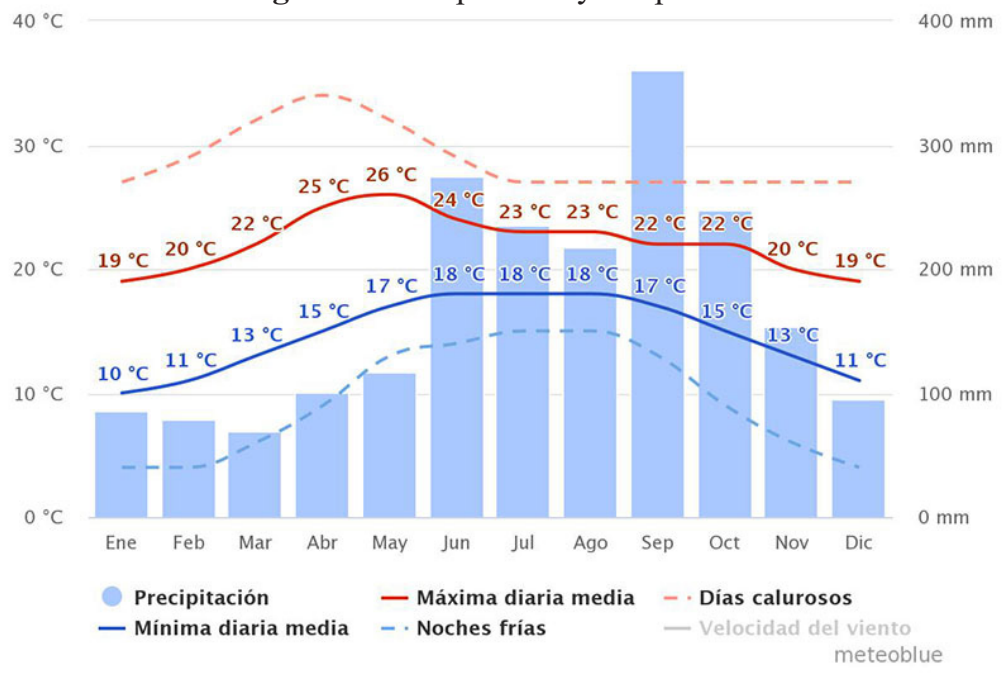

Nota. Retomada de Meteoblue, 2021

\section{El riesgo de inundación}

En la colonia Encinal, se construyeron viviendas en condiciones de irregularidad debido a la baja accesibilidad para adquirir una propiedad en la zona urbana consolidada. En el proceso se utilizaron suelos poco aptos para el desarrollo urbano, se constriñó el arroyo y eliminaron los taludes naturales, y se rellenó el cauce con material orgánico. Además, existe una fuerte contaminación por el depósito de residuos sólidos de las viviendas. 
El arroyo (ahora canal) se modificó para que las zonas habitacionales de los asentamientos urbanos Tezontles, Zaragoza, Arenas, Manantiales y Encinal descargaran las aguas servidas por medio del drenaje y a cielo abierto (ver figura 4). Esta situación preocupa a la población, debido a las enfermedades generadas por dicha contaminación (López, 2021).

El gobierno municipal ha incidido en estas condiciones, debido a la implementación de proyectos de urbanización en el cauce como la red vial, drenaje y alcantarillado, mismas que presentan agrietamientos y hundimientos por las inundaciones (ver figura 5).

En los periodos de mayor precipitación, aumentan los volúmenes de agua en el cauce, existe acumulación de flujos pluviales en las vialidades y colapsa el drenaje, lo que generan inundaciones que alcanzan alturas de hasta $45 \mathrm{~cm}$ en las viviendas. Las viviendas están construidas de materiales endebles como lámina, madera y cartón (ver figura 6).

De izquierda a derecha, Figura 4. Drenaje a cielo abierto; Figura 5. Vialidades construidas sobre el cauce;

Figura 6. Viviendas con rezago
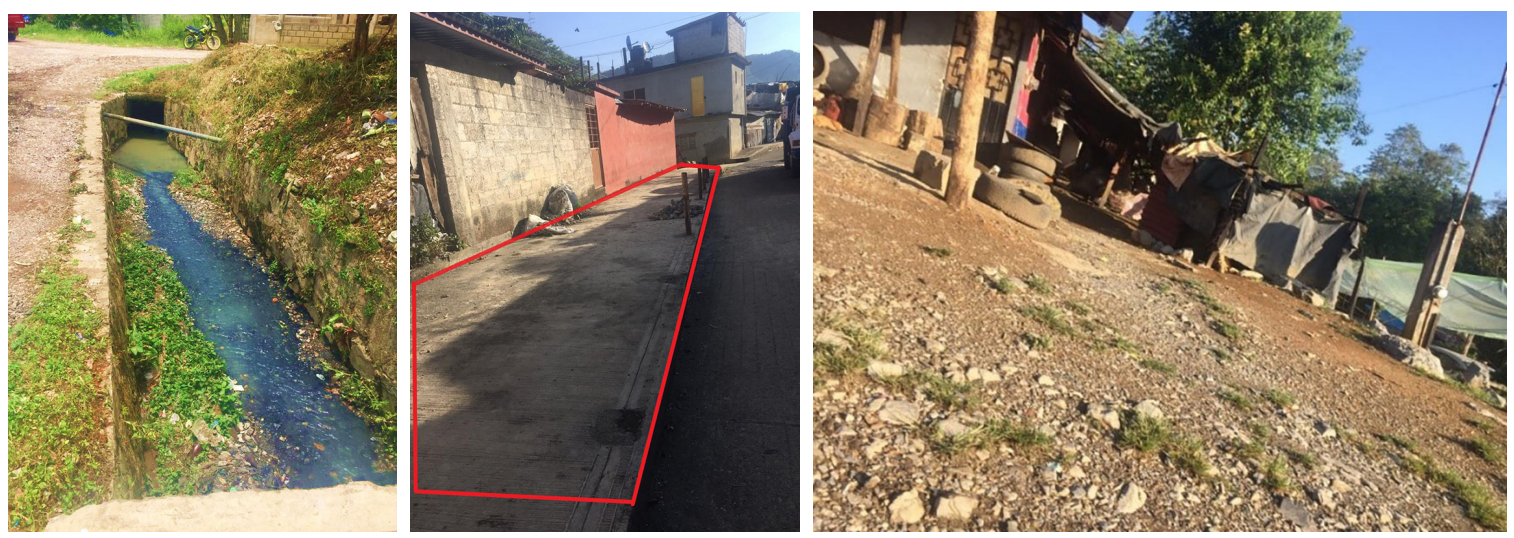

Nota. Propias, tomadas en recorridos a barrios, 2020, 2021

A partir del análisis espacial de los niveles máximos de agua, la categorización de los tramos del arroyo, las altitudes del terreno y las condiciones de las viviendas, se identificaron las manzanas que presentan vulnerabilidad física y social, y se determinaron niveles de riesgo de inundación. En el diagnóstico técnico, sustentado con la percepción social, se identificaron 230 habitantes y 49 viviendas vulnerables (ver figura 7).

Respecto a la política de Gestión Integral de Riesgos, el municipio de Xicotepec cuenta únicamente con el Plan de Desarrollo Municipal (2018-2021), que plantea estrategias de seguridad, educación, salud, economía, desarrollo urbano y rural, sin embargo, no existe una carta urbana que determine y regule los usos de suelo.

Por otro lado, el Atlas de Riesgos del Estado de Puebla (CENAPRED, 2009) es el único estudio de riesgo con el que se cuenta, y si bien contiene diagnósticos y una base de datos geoespacial, estos no permiten identificar el riesgo de inundación en asentamientos urbanos, ni irregulares. En este sentido existe un vacío de dicho instrumento en el nivel municipal. 
Figura 7. Zona de vulnerabilidad

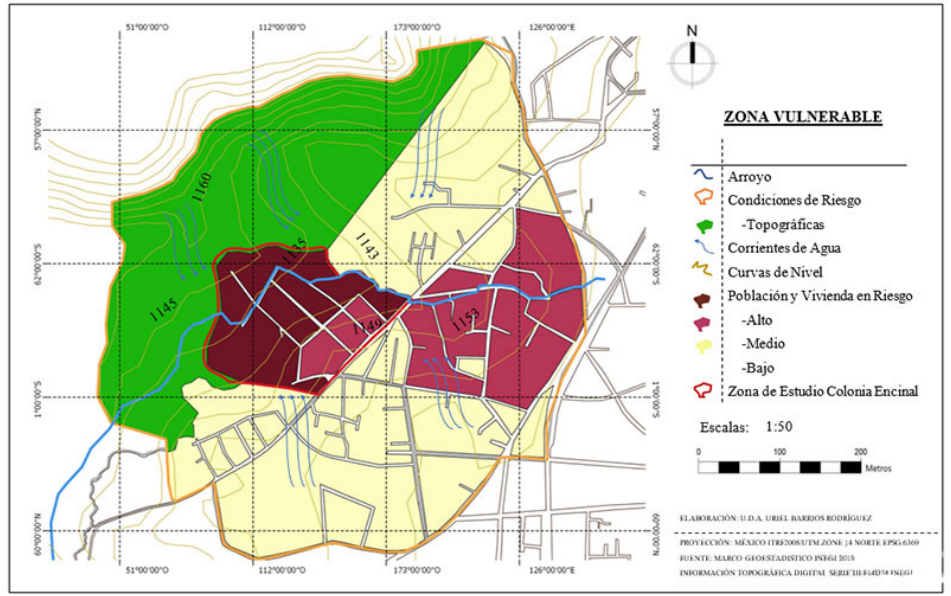

Nota. Elaboración propia

\section{Conclusiones}

La degradación de las condiciones naturales, el desarrollo urbano en zonas inundables, y el rezago son variables que definen la construcción social de riesgo de inundación (Beck, 1998; Merlinsky \& Tobías, 2016). Estas variables permiten establecer relaciones entre los ámbitos social, urbano y ambiental para evidenciar que los riesgos de desastres derivan de las acciones humanas (Lungo, 2005; Lavell, 1996).

Los asentamientos irregulares, como la colonia Encinal, presentan condiciones de precariedad de vivienda, bajo acceso a servicios, e irregularidad que potencian su situación de vulnerabilidad y riesgo ante los impactos de las amenazas naturales y antrópicas.

La carencia de estudios de riesgo en asentamientos urbanos y la subutilización de los Sistemas de Información Geográfica (SIG), particularmente en zonas semirurales como Xicotepec, son debilidades de la política de la Gestión Integral de Riesgos en México, que merman los diagnósticos urbanos y limitan el planteamiento de estrategias y acciones, así como la toma de decisiones.

Existe una baja regulación del uso de suelo en asentamientos en zonas de riesgo, lo que incentiva la apropiación de suelos no aptos para el desarrollo urbano.

La revaloración de la tecnología de los SIG para el análisis espacial de un asentamiento irregular en zona de riesgo de inundación permite generar información específica para determinar las causas naturales y antrópicas que inciden en la generación del riesgo de desastre desde un enfoque complejo.

Este estudio evidencia el riesgo de inundación y vulnerabilidad en un asentamiento irregular, la colonia Encinal, mediante el análisis socioespacial de variables estadísticas y geográficas en formatos vectoriales, raster y alfanuméricos con un software libre, herramientas de geoprocesamiento y modelado.

La investigación contribuye con información respecto a las condiciones de riesgo y vulnerabilidad para concientizar a la población y autoridades gubernamentales en el municipio de Xicotepec, y promover la toma de decisiones concretas para prevenir y mitigar el riesgo de desastre. Además, sirve como referente metodológico para otros casos del municipio de Xicotepec y el Estado de 
Puebla.

\section{Recomendaciones}

- Es necesario realizar investigación-acción mediante métodos de análisis cualitativos y cuantitativos con un enfoque sistémico del riesgo de desastre, en la que se consideren variables naturales, urbanas y sociales para evidenciar las condiciones de vulnerabilidad.

- En los estudios se debe de enfatizar el riesgo como una construcción social derivada, no solo de amenazas de origen natural, sino de múltiples actividades antrópicas que transforman y degradan territorio (Lavell, 1996).

- Se debe resaltar que la percepción del riesgo es en sí una construcción social, culturalmente determinada, que es necesario particularizar en cada territorio (Acosta, 2005).

- Es necesario revalorar los SIG para el estudio de los riesgos de desastre debido a que proporcionan información detallada y permiten definir con mayor precisión las variables sociales y físicas que ayudan a determinar los niveles de vulnerabilidad, principalmente en asentamientos irregulares.

- Es necesario georreferenciar datos en in situ o mediante la percepción remota en asentamientos irregulares, ya que existe un déficit en los instrumentos de planeación y gestión urbana.

- Los instrumentos de planeación deben potencializar los SIG como tecnología para elaborar diagnósticos urbanos y plantear estrategias desde el enfoque preventivo de riesgos en asentamientos urbanos.

\section{Referencias}

Acosta, V. G. (2005). El riesgo como construcción social y la construcción social de riesgos. Desacatos (19), 11-24. http:/www.scielo.org.mx/scielo.php?script=sci_arttext\&pid=S1607-050X2005000300002. ISSN 2448-5144.

Aguilar, A. G., \& Escamilla, I. (2009). Periferia urbana: deterioro ambiental y reestructuración metropolitana. Ciudad de México: Miguel Ángel Porrúa.

Becerra, J. (marzo de 2021). Comunicación personal. (U. Barrios, Entrevistador)

Beck, U. (1998). La lógica del reparto de la riqueza y del reparto de los riesgos. En U. Beck, La sociedad del riesgo, pp.1-80. Barcelona: Paidos.

Buzai, G. (Octubre de 2009). Análisis Espacial con Sistemas de Información Geográfica. Sus cinco conceptos fundamentales. http://www.researchgate.net/publication/298420203_Analisis Espacial_con_Sistemas_de_Informacion_Geografica_Sus_cinco_conceptos_fundamentales

Cardona, A. O. (2003). La necesidad de repensar de manera holística los conceptos de vulnerabilidad $y$ riesgo. Una crítica y una revisión necesaria para la gestión. Obtenido de Red de estudios sociales en prevención de desastres en América Latina: http://www.desenredando.org/public/ articulos/2003/rmhcvr_may-08-2003.pdf 
CENAPRED. (noviembre de 2009). Atlas de Riesgo del Estado de Puebla. (G. d. México, Editor) Recuperado el 2021, de Atlas Nacional de Riesgos: http:/www.atlasnacionalderiesgos.gob. $\mathrm{mx} / \mathrm{app} /$ CoberturaEstatal/

CONEVAL. (2021). Consejo Nacional de Evalución de la Política de Desarrollo Social. Obtenido de https://www.coneval.org.mx/Medicion/IRS/Paginas/Que-es-el-indice-de-rezago-social. aspx

Córtes , F. (2003). Evolución y características de la pobreza en México en la última década del siglo XX. Nueva Época, (2), 295-325.

CPEUM. (2020). Ley de Aguas Nacionales. Última reforma publicada DOF 06-01-2020, Artículo 27 , 07. México: Cámara de Diputados del h. Congreso de la Unión. Obtenido de http://www. diputados.gob.mx/LeyesBiblio/pdf/16_060120.pdf

Díaz Delgado, C., Baro Juárez, J. E., \& Bedolla Lara, S. (2011). Valoración económica de daños directos por inundación en Valle de Chalco Solidaridad, Estado de México. Obtenido de Eventos INEGI: https://www.inegi.org.mx/contenidos/eventos/2011/infogeo/ET4_36_ DIAZ.pdf

Gobierno federal. (2021). Ley General de Protección Civil. México. Recuperado el 2021, de http:// www.diputados.gob.mx/LeyesBiblio/pdf/LGPC_200521.pdf

H. Ayuntamiento. (2018-2021). Plan de Desarrollo Municipal del Municipio de Xicotepec, Puebla. Obtenido de Gobierno de Puebla: https:/ojp.puebla.gob.mx/index.php/zoo-items-landing/ item/plan-de-desarrollo-municipal-del-municipio-de-xicotepec-puebla-2018-2021

Hernández, U. R., Barrios, P. H., \& Ramírez, A. (2017). Análisis de riesgo por inundación: metodología y aplicación a la cuenca Atemajac. Tecnología y ciencia del agua, 8(3), pp. 5-25. https://doi.org/10.24850/j-tyca-2017-03-01

INEGI. (2010). Censo de Población y Vivienda. México. Obtenido de Censo de Población y Vivienda: https:/www.inegi.org.mx/datos/

INEGI. (2016). Servicios. Obtenido de Mapas: https://www.inegi.org.mx/app/mapas/

Laguno, O. J., \& Bourguett, V. (2014). Generación de mapas de riesgo considerando la topografía urbana, aplicado a la ciudad de Oaxaca de Juárez, Oaxaca. Obtenido de Repositorio UNAM: https://repositorio.unam.mx/contenidos/generacion-de-mapas-de-riesgo-considerando-la-topografia-urbana-aplicado-a-la-ciudad-de-oaxaca-de-juarez-oaxaca-73681?c=BZPzmG\&d=false \&q=*:*\&i=1\&v=1\&t=search_0\&as=0

Lavell, A. (1996). Degradación Ambiental, Riesgo y Desastre Urbano. Problemas y Conceptos: Hacia la Definición de una Agenda de Investigación. (M. A. Fernández, Productor) Obtenido de LA RED: https://www.desenredando.org/public/libros/1996/cer/CER_cap02-DARDU_ ene-7-2003.pdf

López, T. (marzo de 2021). Comunicación personal. (U. Barrios, Entrevistador)

Lungo, M. (2005). Riesgos urbanos. (itsmo, Ed.) San Salvador: DOE-UCA.

Merlinsky, M. G., \& Tobías, M. A. (2016). Inundaciones y construcción social del riesgo en Buenos Aires. Acciones colectivas, constroversias y escenarios futuros. Cuaderno del Cendés, 33(91), 
45-63. Recuperado el septiembre de 2021, de http://ve.scielo.org/scielo.php?script=sci_arttext\&pid=S1012-25082016000100004\&lng=es\&nrm=iso

Meteoblue. (2021). Clima de Xicotepec de Juárez. Obtenido de https:/www.meteoblue.com/ es/tiempo/historyclimate/climatemodelled/xicotepec-de-ju\%c3\%a1rez_m\%c3\%a9xico_3514510

Moe, T. L., \& Pathranarakul, P. (2006). An integrated approach to natural disaster management: Public projectmanagementanditscriticalsuccesfactors. DisasterPrevention and Management: An International Journal, 15(3), 396 - 413. http://dx.doi.org/10.1108/09653560610669882

Mojtahedi, M., \& Oo, B. L. (2016). Critical attributes for proactive engagement of stakeholders in disaster risk. International Journal of Disaster Risk Reduction, 35-43. https://doi. org/10.1016/j.ijdrr.2016.10.017

Narvaéz, Q. I., Durán, S. G., Menoscal, C. J., \& Bayón, J. M. (2020). Espacio urbano periférico y la construcción social del riesgo en ciudades inermedias. Cuadernos de vivienda y urbanismo, 13, 1-18. https://doi.org/10.11144/Javeriana.cvu13.eupc

Narvéz, L., Lavell, A., \& Pérez, G. (2009). La Gestión del Riesgo de Desastre. Un enfoque basado en procesos. Obtenido de https://www.eird.org/cd/herramientas-recursos-educacion-gestion-riesgo/pdf/spa/doc17733/doc17733-a.pdf

Omelio, C. R. (2012). Estudios de peligros, vulnerabilidad y riesgos de desastres en Cuba. REDVET. Revista Electrónica de Veterinaria, 13(05), 1-16. E-ISSN: 1695-7504

ONU. (1987). El Decenio Internacional para la Reducción de los Desastres. Obtenido de https://iris.paho.org/bitstream/handle/10665.2/16777/v108n3p260.pdf?sequence=1\&isAllowed=y\#: :text=La\%20Resoluci\%C3\%B3n\%20169\%20de\%201a,Reducci\%C3\%B3n\%20 de $\% 201$ os $\% 20$ Desastres $\% 20$ Naturales.\&text $=$ En $\% 201$ os $\% 20 \%$ C3\%BAltimos $\% 20 d$ decenios $\% 20$ pueden $\% 20$ haber $\% 20$ causado $\% 2$

ONU. (2015). Marco de Sendai para la Reducción del Desastre 2015-2030. Recuperado el Agosto de 2021, de UNISDR: https://www.unisdr.org/files/43291_spanishsendaiframeworkfordisasterri.pdf

Pelling, M. (2005). Measuring vulnerability to urban disaster risk. Open House International on managing urban disasters,(1), 125-132.

Plot, B. (2011). Vulnerabilidad en los asentamientos irregulares del amba: analisis de caso: Barrio Sarmiento. Revista Geográfica de América Central, (2), 1-17. https://www.revistas.una.ac.cr/ index.php/geografica/article/view/252

Principi, N., \& Buzai, G. D. (2021). Mapa social y vulnerabilidad socioeconómica en la ciudad de Luján, Buenos Aires, Argentina. En S. S. Villerías, G. Nochebuena, \& C. d. Acapulco (Ed.), Procesos territoriales un enfoque multidisciplinario. Lugar Editorial.

Radicelli, C., Pomboza, M., Villacrés, P., \& Bodero, E. (2019). Sistemas de información geográfica y su aplicación en las Ciencias Sociales. Chakiñan: Revista de Ciencias Sociales y Humanidades, (8), 24-35. Obtenido de https://www.redalyc.org/journal/5717/571760747003/html/

Ramírez, F. J. (enero de 2017). Gestión para la Reducción del Riesgo de Inundación en el Municipio de Puebla. BUAP. Recuperado el 2021, de https://repositorioinstitucional.buap. 
$\mathrm{mx} / \mathrm{handle} / 20.500 .12371 / 1118$

Ríos-Llamas, C. (Enero-Junio de 2020). Fragilidad urbana: una lectura del desastre desde los habitantes menos protegidos. Quivera Revista de Estudios Territoriales(22), 57-74. https:// doi.org/10.36677/qret.v22i1.12798

Rodríguez, H. G., \& García, N. H. (2012). Inundaciones en zonas urbanas. Medidas preventivas $y$ corretivas, acciones estructurales y no estructurales. Obtenido de Programa de maestría y doctorado: http://www.ptolomeo.unam.mx:8080/xmlui/bitstream/handle/132.248.52.100/5281/Tesis.pdf?sequence $=1$

Ruiz, V., Savé, R., \& Herrera, A. (2013). Análisis multitemporal del cambio de uso de suelo, en el Paisaje Terrestre Protegido Miraflor Moropotente Nicaragua, 1993-2011. ecosistemas, 117-123.

Salas, O. (2019). ¡Fuerte huracán azota Xicotepec! . Cambio, págs. 08-09.

Salgado, M. S. (noviembre de 2020). Empoderamiento ciudadano para mitigar el riesgo de inundación. Caso La Hacienda, Puebla. Obtenido de Repositorio Institucional BUAP: https://repositorioinstitucional.buap.mx/handle/20.500.12371/10572

SMN. (2019). Sistema Meteorológico Nacional. Obtenido de Normales climatológicas: https:// smn.conagua.gob.mx/es/

Vázquez-Sanchez, M., \& Méndez-Ramírez, J. J. (enero-junio de 2011). La vulnerabilidad de los asentamientos en espacios no urbanizables en el muncipio de San Mateo Atenco, Estado de México. Quivera. Revista de Estudios Territoriales, 13(1), 244-268. Obtenido de https:// www.redalyc.org/articulo.oa? id=40118420015

Wilches-Chaux, G. (1993). La vulnerabilidad global. En A. Maskrey, Los desastres no son naturales. 11-41: LA RED. Recuperado el Agosto de 2021, de https://www.desenredando.org/public/ libros/1993/ldnsn/LosDesastresNoSonNaturales-1.0.0.pdf

Yamanaka Victor H, A. (2011). Construcción de mapas de riesgo de inundación en ríos en la zona del Soconusco. Obtenido de Ponencias tercer seminario Victor Alcocer: https://imta.gob.mx/ potamologia/images/stories/ponencias_tercer_seminario/seminario/victor_alcocer/mapasriesgosdeinundacion.pdf 